\title{
Semantic and syntactic demarcations of Classical Greek object cases: An object(ive) study
}

\author{
AJ Murphy, Stanley Dubinsky \& Mark Beck*
}

\begin{abstract}
In Classical Greek, many verbs take direct objects marked with genitive (GEN) or dative (DAT), rather than accusative (ACC) case. Traditional grammars (Smyth 1956, Boas et al. 2019) fail to offer principled descriptions or accounts of the distribution of ACC, GEN, DAT object case for transitive verbs. This paper analyzes a corpus involving case-assigning transitive verbs, and examines Luraghi's 2010 Transitivity Hierarchy in this context. We find that, while her ranking of verbs' transitivity is correct, the features used to determine the hierarchy are not. Our study demonstrates a highly significant correlation between a verb's level of transitivity (as indicated by the case marking on its object) and the Proto-role Properties of Change of State and subject Volitionality (Dowty 1991).
\end{abstract}

Keywords. syntax; semantics; Classical Greek; case marking

1. Introduction. Standard syntactic accounts of Accusative case-marked NP verb complements treat them, unremarkably, as direct objects (DO), but treat Dative and Genitive marked complements as if they occupy a secondary, indirect object (IO) role (Chomsky 1986). From this perspective, the behavior of Dative and Genitive NP complements of mono-transitive verbs in languages like Classical Greek (CG) is confounding. CG productively places DAT and GEN NPs as the singular complement to many monotransitive verbs, allowing them to occupy what would otherwise be thought of as the direct-object position. Not only is there a class of verbs in CG which productively take GEN and DAT direct objects, but there is also a class of verbs which may optionally alternate between ACC and GEN. Therefore, the attested verb-classes are as follows: i) ACC-Object verbs, ii) GEN-Object verbs, iii) DAT-Object verbs, and iv) ACC/GENObject verbs. The present research investigates this phenomenon through a corpus-based analysis that seeks to uncover the statistical tendencies of the nuances of DO Cases in terms of the verbs that select them.

In brief, the present study confirms that Luraghi's (2010) Transitivity Hierarchy makes correct predictions regarding the case-marking of objects, such that objects of highly transitive verbs get accusative case, medium transitive verbs get genitive case, and low transitive verbs get dative case. However, the present data illuminate flaws in the formalization of the Hierarchy, in that Luraghi's Hierarchy only considers the semantic properties of case-marked objects, and thereby fails to recognize all the feature that determine this heirarchy. We find that better results are obtained when one examines the semantic properties of both objects and subjects. In testing Dowty's (1991) Agent and Patient proto-roles, it becomes clear that the Agent Proto-role of Volitionality better predicts transitivity than does the Patient Proto-role of Affectedness. In accurately accounting for the semantic properties that explain levels of transitivity, the present study resolves a long-standing puzzle regarding the assignment of genitive and dative case to direct objects of monotransitive verbs, providing a more consistent analysis than that which is

\footnotetext{
${ }^{*}$ We would like to thank the following individuals for their helpful input at the 2020 LSA Conference: Peter Cullicover, Brian Joseph, and Elitzur Bar-Asher Siegal. We would also like to thank Amada Dalola for her kind and valuable guidance on the statistical approach used within this study. All errors and omissions are exclusively our own.

Authors: AJ Murphy, University of South Carolina (ajmurphy@email.sc.edu), Stanley Dubinsky, University of South Carolina (dubinsk@mailbox.sc.edu), \& Mark Beck, University of South Carolina (beckma@mailbox.sc.edu).
} 
typically given, namely that genitive-marked objects are partitive (Hansen \& Quinn 1992, Boas et al. 2019), which is an analysis that also ignores the systematic assignment of dative-marked direct objects.

Section 1 reviews prior work on this topic, primarily that of Luraghi $(2003,2010)$, and discusses some of the shortcomings in that literature. Section 2 discusses the usefulness of Dowty's (1991) conception of Proto-Roles, particularly in terms of the goals of the present study. Section 3 then presents the data domain and methodology that figure in our study, and section 4 describes some preliminary (pre-statistical) observations that come to the fore. In section 5, we present the statistical results of our examination of the distribution of ACC, GEN, and DAT case-marking on objects - first under the lens of Luraghi's featural analysis of these, and then using a revised set of features that yields superior results. Finally, Section 6 provides a discussion of the above, along with some conclusions and directions for future research.

2. Previous treatments of Classical Greek case. Outside of Classical Greek pedagogical grammars, there have not been many linguistic treatments of case assignment or argument structure in CG, particularly in reference to the atypical behavior of dative and genitive direct objects. A largely general description of the distribution of ACC, DAT, and GEN case is provided in Luraghi 2003, and a somewhat more specific description of their distribution as casemarking on direct objects, equating this with semantic "levels of transitivity" in Luraghi 2010.

According to Luraghi (2010), ACC objects (whose semantic role is characterized as "theme") are "wholly affected patients, which undergo a change of state" (p. 64) and which canonically occur with the most highly transitive verbs. It is claimed that ACC case is nearly always assigned in such highly transitive contexts, and that GEN case is exceptionally assigned to objects having a partitive interpretation. In Luraghi's terms, GEN objects that are complements of highly transitive verbs are "partitive genitive" and seen to be less affected (although still "somewhat" affected) than are ACC objects. This analysis mirrors the explanation put forth by most pedagogical grammars, claiming that GEN objects are partitives and are relics of GEN indirect objecthood (Hansen \& Quinn 1992, Boas et al. 2019). Verbs that are subcategorized for DAT objects are, according to Luraghi, even less transitive than those that are subcategorized for ACC or GEN objects.

The proposed Transitivity Hierarchy, intended to account for the mapping of verb meaning to case, distinguishes three levels of transitivity: High transitivity (HT), medium transitivity (MT), and low transitivity (LT). These levels are distinguished on basis of (i) whether the verb's object undergoes a Change of State (COS), and (ii) whether the object is affected.

\begin{tabular}{lccl} 
& $\begin{array}{c}\text { Change of State } \\
\text { (COS) }\end{array}$ & Affect & Case-assignment \\
\hline High Transitivity & + & + & ACC \\
Medium Transitivity & - & + & GEN \\
Low Transitivity & - & - & DAT \\
\hline
\end{tabular}

Table 1. Luraghi’s (2010) Transitivity Hierarchy

While Luraghi provides a worthy point of entry into the topic, the analysis is weakened by certain methodological issues which the present research aims to correct. First, Luraghi (2003/2010) presents an analysis of Ancient Greek (AG), a corpus spanning some 1200 years (encompassing the period from $900 \mathrm{BCE}$ to $500 \mathrm{CE}$ ). Accordingly, the analysis utilizes data from all of Homeric Greek ( 800 BCE), Classical Greek ( 500-300 BCE), and Koine Greek ( 300 
BCE-400 CE), thereby trying to capture generalizations about varieties of Greek that are separated by many centuries, if not a millennium. Utilizing a range of data that is diachronically distant as is Old English and Modern English runs the risk of conflating meaning-case correlations that may not be consistent across time. By restricting our study to consider only Classical Greek texts, we carefully examine a corpus spanning only 2 centuries ( $500-300$ BCE), which is likely to be systematically more consistent.

Secondly, the data brought to bear in this analysis are limited to some 20 examples of casemarking verbs, suggesting (if not representative of a much larger data collection) that the observations and analysis are rather tentative and preliminary. In our study here, we present a description of case marking that considers nearly 200 sentences, representing examples of some 25 verbs.

Finally, where Luraghi 2010 only considers two semantic properties of direct objects in framing the Transitivity Hierarchy (COS and Affectedness), the present study expands the range of semantic properties considered analysis by assessing the relevance of all 10 Subject and Object Proto-role properties proposed in Dowty 1991 to the matter of transitivity.

3. Argument structure and thematic roles. Thematic roles ( $\theta$-roles) have been treated as useful features by which to describe and organize the relationships between the semantic and syntactic properties of a verb's arguments (i.e. its subject and objects). Traditionally, these include roles such as Agent, Patient, Goal, Source, Experiencer, and Theme, among others. $\theta$ roles are assumed to be assigned to argument positions and provide semantic interpretation of the relation between a verb and its arguments, as well as to determine the argument structure projected and cases assigned by any given verb. The use of a fixed inventory of $\theta$-roles as an explanatory device has come under some criticism in the past (Jackendoff 1987, Dowty 1991).

Dowty 1991, in particular, takes issue with their existence as unanalyzable units of analysis, proposing instead a group of semantic properties that serve as 'tendency-makers' which map a verb's arguments into subject or object grammatical slots based on the collective weight of those properties, as exhibited by any given argument.

The semantic properties that Dowty proposes are divided into two major classes: i) Proto-agent properties and (ii) Proto-patient properties, wherein arguments having Protoagent properties tend to be mapped into subject positions and arguments having Proto-patient properties tend to be mapped into object positions. The individual Proto-roles are grouped as follows under the two headings shown below in Table $2:{ }^{1}$

\footnotetext{
${ }^{1}$ While some of Dowty's 1991 Proto-Roles are self-explanatory and some are less so, it is beyond the scope of this discussion to present a detailed description of them, and it is also the case that the roles which are relevant to the analysis presented here, $\operatorname{COS}$ (i.c/ii.a) and Volitionality (i.a), are quite transparently and intuitively understandable.
} 
i. Dowty's Proto-Agent roles:

a. Volitional involvement in the event or state

b. Sentience (and/or perception)

c. Causing an event or change of state in another participant

d. Movement (relative to the position of another participant)

e. Exists independently of the event named by the verb

ii. Dowty's Proto-Patient roles:

a. Undergoes change of state

b. Incremental theme

c. Causally affected by another participant

d. Stationary relative to movement of another participant

e. Does not exist independently of the event, or not at all

Table 2. Dowty's Proto-role properties ～(Dowty 1991: 572-574)

The actual mapping of arguments to grammatical functions, utilizing these Proto-roles, is actually handled by an Argument Selection Principle (Dowty 1991: 576), by the application of which "the argument for which the predicate entails the greatest number of Proto-Agent properties will be lexicalized as the subject of the predicate; the argument having the greatest number of Proto-Patient entailments will be lexicalized as the direct object". We would contend that Dowty's more nuanced approach to the mapping of arguments to subject and object functions is useful in shedding light on the case marking observed on the objects of CG verbs, which have so far resisted traditional analysis.

4. Data and methodology. While previous considerations of CG case have attempted to provide answers that generalize across all of Ancient Greek, the present study concerns itself only with Classical Greek (c. 500-300 BC). By virtue of this constraint, we are able to examine a data domain that is internally consistent and avoiding possibly confounding semantic changes that might arise over a longer time span. Our study also considers only works of prose, excluding poetic and theatrical works, so as to avoid the inherent anomalies that arise in works stylistically constrained by meter (which often involve, for this reason, violations of canonical grammatical form). For example, in poetry (but not prose) it is not uncommon for an author to choose a nontraditional DO case if that case, for being syllabically longer/shorter or containing a long/short vowel, better fit the metric flow of the construction. Prose (e.g., historical accounts and rhetorical works) is not so grammatically fluid and therefore offers more reliable indicators of DO casemarking parameters of CG. The following authors then make up the source material for the present study: Herodotus (c. 484-425 BC), Thucydides (c. 460-400 BC), Lysias (c. 445-380 BC), Isocrates (c. 436-338 BC). Xenophon (c. 431-354 BC), Plato (c. 428-348 BC), Aeschines (c.389314 BC), Demosthenes (c. 384-322 BC), and Aristotle (c. 384-322 BC).

In order to avoid potential confounds inherent in semantically and grammatically anomalous verb phrases, the data examined was limited to sentences containing monotransitive predicates in active constructions with a single direct object complement. Accordingly, psychological predicates were disregarded, as the subject-object relationship of verbal complements in these constructions tends to be more fluid, as in example (1). Filip (1996: 3) asserts, apropos of this concern, "linking in the domain of psychological predicates is problematic if we assume that there is a direct and uniform association between thematic (or lexical semantic) arguments and syntactic arguments". 
a. Snakes frightened Sam.

b. Sam feared snakes.

Propositional attitude verbs were also disregarded, as such predicates take (typically clausal) propositions rather than direct object NPs as complements, and object case is not clearly assigned by the propositional attitude verb to its complement, as in example (2).

(2) Tom believes [Sam fears snakes].

4.1. Data collection. Data was collected using Tufts University's Perseus Online Digital Library, a corpus of 13,507,448 Ancient Greek words in 2,908 Ancient Greek works. Each verb identified as selecting an ACC, GEN, or DAT-marked direct object was searched for in all of its possible forms (i.e. in all six principal parts and inflections). Instances of sentences containing target verbs that fit our data constraints were located in their original CG and then translated into English. Thirty-one verbs were initially identified, six of which were excluded for a lack of tokens, so that 195 instances across twenty-five verbs were collected.

4.2. DATA ANALYSIS. Under the present analysis, data was analyzed both descriptively and statistically. Collected tokens were coded according to all of Dowty's Proto-Role Properties, as well as according to Luraghi's Transitivity Hierarchy, and again for the Revised Hierarchy that we will posit in section 6.2 .

The present study investigates which factors affect the assignment of the three different Object Case-markers, ACC, GEN, and DAT. Accordingly, the present data was coded such that the dependent variable, Case-marking, was coded as either ACC, GEN, or DAT. While the class of verbs which alternate between ACC/GEN have been previously mentioned, it is the task of the present study to investigate the first three, more traditional, verb-classes.

The data was examined utilizing Dowty's (1991) inventory of Proto-Role Properties, in order to determine whether Luraghi's (2010) transitivity hierarchy would be attested by the data or need to be revised. By invoking Dowty's inventory of subject and object semantic properties, we aimed to test the predictive capacity of these with respect to correlations between the semantic nature of verbs and their assignment of case to objects.

Each verb-token was coded as +/- for Dowty's Agent Proto-Role features as indicated by their subjects: undergoing a change of state, being volitional, movement, independent existence, and sentience to the event. Each verb-token was also coded as +/- for each of Dowty's Patient Proto-Role features as indicated by their direct objects: undergoing a change of state, incremental theme, being causally affected, movement, and dependent existence. Utilizing the two direct object semantic features (COS and Affectedness) which determine a verb's level of transitivity according to Luraghi's analysis, the transitivity of each monotransitive construction was calculated, such that (+COS, +Affected) instances were rated high transitivity, (-COS, + Affected) instances were rated medium transitivity, and (-COS, -Affected) rated low. The results of this analysis are provided in section 5.1 below. Then, after this initial analysis, the entire corpus of constructions was examined to independently assess the distribution of each according to the full range of object and subject semantic proto-role properties. From this examination, it was found that the subject/object feature COS and the subject feature Volitionality were both significant in regard to the assignment of object case. The transitivity of each monotransitive construction was then recalculated, such that $(+\mathrm{COS},+$ Volitional) instances were rated high transitivity, $(-\mathrm{COS},+$ Volitional) instances were rated medium transitivity, and (-COS, - Volitional) rated low, as described below in section 6.2. 
5. Preliminary observations. Here our preliminary observations note descriptive trends in the data which we noted before running any statistical analysis. In our data, ACC case-marking verbs typically denote events that are telic and which affect the object. Such verbs focus on events wherein there is a clear distinction between the before and after (highly telic, and a clear affect). The subjects and object of these verbs consistently align with Dowty's Proto-roles, such that their subjects consistently have Proto-Agent Properties, and their objects consistently have Proto-Patient Properties. This observation is consistent with descriptions given in Luraghi 2003 and 2010, and are consistent with our typical understanding of "theme" and the canonical direct object semantic role, as in example (3) illustrating the ACC case-marking verb, apekteinan 'kill'.

(3) tous ortug-as kai tous alektruon-as $\quad$ k $\quad$ apektein-an

the quails-ACC.M.PL and the roosters-ACC.M.PL kill.AOR.IND.ACT-3PL

'They killed the quails and the roosters.' (Aeschines, Against Timarchus 1.59)

$\mathrm{GEN}^{2}$ case-marking verbs typically denote states or activities (i.e., are atelic). Their effect on the object is not always clear or important to the action described. Often the effect on the subject is more important and focused than the effect on the GEN object. The arguments of GEN case-marking verbs are much less consistent than ACC case-marking verbs with respect to their having canonical subject and object Proto-role Properties. An example of a GEN case-marking verb, amelein 'neglect', is shown in (4).

(4) tois de spousaiois oukh oion te tēs aretēs amel-ein the but earnest.DAT.M.PL not such CONJ the virtue.GEN.F.SG neglect.PRES-INF 'But the good cannot neglect such virtue.' (Isocrates, To Demonicus 1.48)

DAT case-marking verbs typically denote events that are directed towards the object, although the effect on the object is often not clearly realized. The arguments of DAT casemarking verbs are, like GEN case-marking verbs, less consistent than ACC case-marking verbs with respect to their having canonical subject and object Proto-role Properties. An example of a DAT case-marking verb, apeilēsas 'threaten', is shown in (5).

(5) polla apeilēs-as tois Khi-ois mighty.ADV threaten.AOR.IND.ACT-2SG the Chians-DAT.M.PL 'Mightily you threatened the Chians.' (Thucidydes, The Peloponnesian War 8.33)

6. Results. This section presents the results of two analyses of object case-marking, first testing the correlation of Luraghi's proposed features Change of State (COS) and Affectedness with her proposed Transitivity Hierarchy (section 5.1), and next testing a wider range of Proto-role Properties for their correlations with transitivity (section 5.2).

6.1. EXAMINING OBJECT PROPERTIES. Counts of the data indicate that Dowty's Patient Proto-roles of Change of State (COS) and Affectedness correlate with differences in the case-marking of objects. In contrast, the Patient Proto-roles of Independent Existence, Movement, and Incremental Theme played no distinguishable role in classifying verbs that variously mark their objects with ACC, GEN, or DAT case.

\footnotetext{
${ }^{2}$ It was suggested to us during LSA 2020 that perhaps the Genitive of Negation in Russian could shed light on the assignment of Genitive in Classical Greek. While we did look through our data with this perspective considered, our descriptive analysis noted no uniformity in the negation of a proposition and the subsequent case-marking of ACC, GEN, or DAT, and was therefore not considered a meaningful variable under our stastical analysis.
} 
Considering the Patient Proto-roles of objects alone, it would appear that COS and Affectedness correlate with ACC/GEN/DAT case marking choices, and indeed Luraghi's analysis, focusing as it does on these properties of the object to the exclusion of subject properties, builds its transitivity hierarchy utilizing these two features. The rows in Table 3 are arranged according to Luraghi's Hierarchy, with ACC being highest transitivity, GEN being next, and DAT being last. The columns in Table 3 are also arranged according to Luraghi's Hierarchy, (+COS, +Affected) being most transitive, (-COS, +Affected) being next, and (-COS, -Affected) being last.

If Luraghi's hierarchy and the features that determine it are correct, then the number of instances of ACC-case verb constructions should be highest in the first column under $(+\mathrm{COS}$, +Affected), the number of instances of GEN-case verb constructions should be highest in the second column under (-COS, +Affected), and the number of instances of DAT-case verb constructions should be highest in the last column under (-COS, -Affected). What we find, however, does not confirm Luraghi's predictions. While the largest number of ACC-case verb constructions are indeed in the first column $(n=30)$, the largest number of GEN-case verb constructions are split across columns two $(n=23)$ and three $(n=24)$, and the largest number of DAT-case verb constructions are found in column $\operatorname{two}^{3}(n=49)$.

\begin{tabular}{llll} 
& $\begin{array}{l}\text { High } \\
(+\mathrm{COS},+ \text { Affected })\end{array}$ & $\begin{array}{l}\text { Medium } \\
(-\mathrm{COS},+ \text { Affected })\end{array}$ & $\begin{array}{l}\text { Low } \\
(-\mathrm{COS},- \text { Affected })\end{array}$ \\
\hline ACC & $\mathbf{3 0}$ & 8 & 0 \\
GEN & 1 & $\mathbf{2 3}$ & $\mathbf{2 4}$ \\
DAT & 2 & $\mathbf{4 9}$ & 4
\end{tabular}

Table 3. Results utilizing Luraghi's Transitivity Hierarchy (COS and Affectedness)

As Table 4 below shows, objects of ACC case-assigning verbs uniformly undergo COS, whereas objects of GEN and DAT case-assigning verbs do not. Objects of ACC and DAT case-assigning verbs are uniformly causally affected, but objects of GEN case-assigning verbs are not consistently so.

\begin{tabular}{lll} 
& COS (undergo change of state) & Causally Affected \\
\hline ACC-marking verb & YES & YES \\
GEN-marking verb & NO & SOMETIMES \\
DAT-marking verb & NO & YES \\
\hline
\end{tabular}

Table 4. Corellation of Patient Proto-roles and case

Reexamining Luraghi's transitivity hierarchy, we find that her predictions are not borne out by the observed data, in that objects of GEN case-assigning verbs are not consistently Affected (contra Luraghi). Furthermore, as we will demonstrate later, Affectedness turns out not to be a usefully clear or consistent property to account for the mapping from meaning to case.

Our descriptive assessment of this data is confirmed through statistical analysis. A Chisquare analysis reveals a highly significant interaction between the features of COS and Affectedness and the assignment of case $([\mathrm{X} 2(4,141)=121.82, \mathrm{p}<.0001])$. However, while these

\footnotetext{
${ }^{3}$ Thank you to Elitzur Bar-Asher Siegal for his input on the particulars of the behavior of DAT as unformly signalling affectedness in Classical Greek, as well as Modern Hebrew (Bar-asher Siegal \& Boneh 2015), which is supported by the results presented herein.
} 
two properties do indeed form the basis of Luraghi's Transitivity Hierarchy, the Chi-Square residuals (Table 5) contradict Luraghi's claim that GEN objects are more transitive than DAT objects. Thus, while the interaction is highly significant, the results show DAT case-assigning verbs to be more transitive than GEN case-assigning verbs, contrary to the ranking proposed in Luraghi 2010. Thus, either her ranking is wrong, or the properties used to determine that ranking are so. The balloon plot of the residuals (Figure 1) further illustrates this problem with Luraghi's predictions.

\begin{tabular}{llll} 
& ACC & GEN & DAT \\
\hline High & $\mathbf{7 . 0 7 7}$ & -3.053 & -3.030 \\
Medium & -2.920 & -0.811 & $\mathbf{3 . 1 8 5}$ \\
Low & -2.747 & $\mathbf{4 . 6 8 6}$ & -2.094 \\
\hline
\end{tabular}

Table 5. Chi-Square Pearson's residuals (Luraghi’s Transitivity Hierarchy)

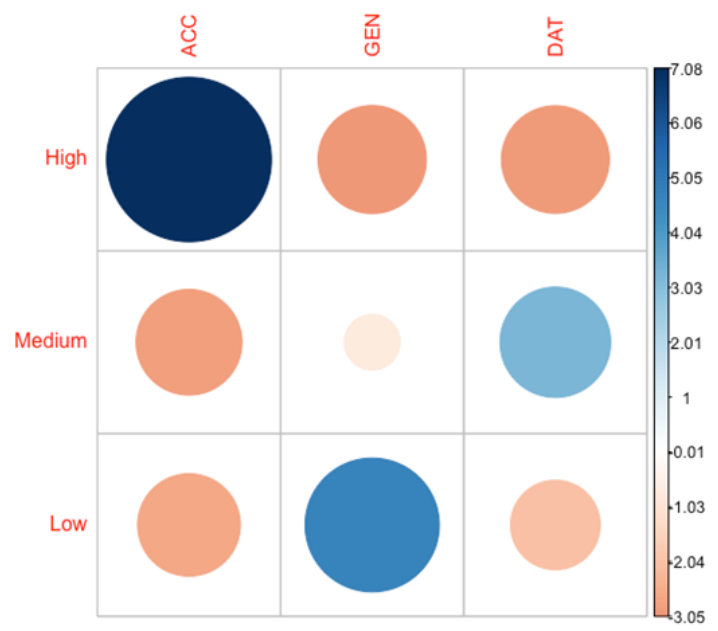

Figure 1. Balloon plot of Chi-Square residuals (Luraghi's Transitivity Hierarchy)

6.2. EXAMINING SUBJECT PROPERTIES. This section demonstrates the relevance of Dowty's Agent Proto-roles (in addition to the Patient Proto-roles) in accounting for the distribution of object case-marking respective of verb meaning. As the results presented in Section 5.1 make clear, if Luraghi's features are correct, then her hierarchy cannot be. And vice versa. By including the metric of Agent Proto-roles in assessing this distribution, we find that the Agent/Patient Protorole COS (as before), together with the Agent Proto-role Volitionality, strongly correlate with the differential case-marking of objects. On the other hand, the Agent Proto-roles Movement, Independent Existence, and Sentience play no role in distinguishing among verbs that case-mark their objects with ACC, GEN, or DAT.

Here below in Table 6, once again, the rows are arranged according to Luraghi's Hierarchy, with ACC being highest transitivity, GEN being next, and DAT being last. However, the columns are arranged according to the two Agent/Patient and Agent properties (COS and Volitional) for which we found a strong correlation. Here, (+COS, +Volitional) is most transitive, $(-\mathrm{COS},+$ Volitional $)$ is next, and $(-\mathrm{COS},-$ Volitional $)$ is last. In Table 6 , we see that the largest number of ACC-case verb constructions are in the first column $(n=29)$, the largest 
number of GEN-case verb constructions are in the second column $(n=47)$, and the number of DAT-case verb construction is split across columns two $(n=29)$ and three $(n=24)$.

\begin{tabular}{llll} 
& $\begin{array}{l}\text { High } \\
(+ \text { COS },+ \text { Volitional })\end{array}$ & $\begin{array}{l}\text { Medium } \\
(-C O S,+ \text { Volitional })\end{array}$ & $\begin{array}{l}\text { Low } \\
(-C O S,- \text { Volition })\end{array}$ \\
\hline ACC & $\mathbf{2 9}$ & 9 & 0 \\
GEN & 1 & $\mathbf{4 7}$ & 0 \\
DAT & 2 & $\mathbf{2 9}$ & $\mathbf{2 4}$ \\
\hline
\end{tabular}

Table 6. Transitivity Hierarchy results utilizing revised features (COS and Volitionality)

As Table 7 below shows, ACC case-assigning verbs are uniformly associated with COS meanings, but GEN and DAT case-assigning verbs are not. Additionally, subjects of ACC and GEN case-assigning verbs are Volitional, while the subjects of DAT case-assigning verbs only sometimes are.

\begin{tabular}{lll} 
& COS (cause a change of state) & Volitional \\
\hline ACC-verb & YES & YES \\
GEN-verb & NO & YES \\
DAT-verb & NO & SOMETIMES \\
\hline
\end{tabular}

Table 7: Corellation of Agent Proto-Roles and case

Our observations reported above are confirmed through statistical analysis. A Chi-square analysis reveals a highly significant interaction between the features of COS and Volitionality and the assignment of case $([X 2(4,141)=123.7 .82, \mathrm{p}<.0001])$. In Table 8 , we can see from the Chi-Square residuals that these two properties do confirm the distribution of case-marking in accordance with Luraghi's original hierarchy. This interaction shows ACC case-assigning verbs to be the most transitive, with GEN case-assigning verbs being next most transtive, and DAT case-assigning verbs being least transitive, just as proposed in Luraghi 2010. Our results confirm that Luraghi's Transitivity Hierarchy is essentially correct (with ACC > GEN > DAT), but that the relevant properties are in fact Volitionality of Subjects and COS, rather than Affectedness of Objects and COS. The balloon plot of the residuals (Figure 2) further illustrates this relationship.

\begin{tabular}{cccc} 
& ACC & GEN & DAT \\
\hline High & $\mathbf{6 . 9 3 8}$ & -2.998 & -2.967 \\
Medium & -2.906 & $\mathbf{3 . 3 5 8}$ & -0.722 \\
Low & -2.543 & -2.858 & $\mathbf{4 . 7 8 4}$ \\
\hline
\end{tabular}

Table 8: Chi-Square Pearson's Residuals (Transitivity Hierarchy, Revised Features) 


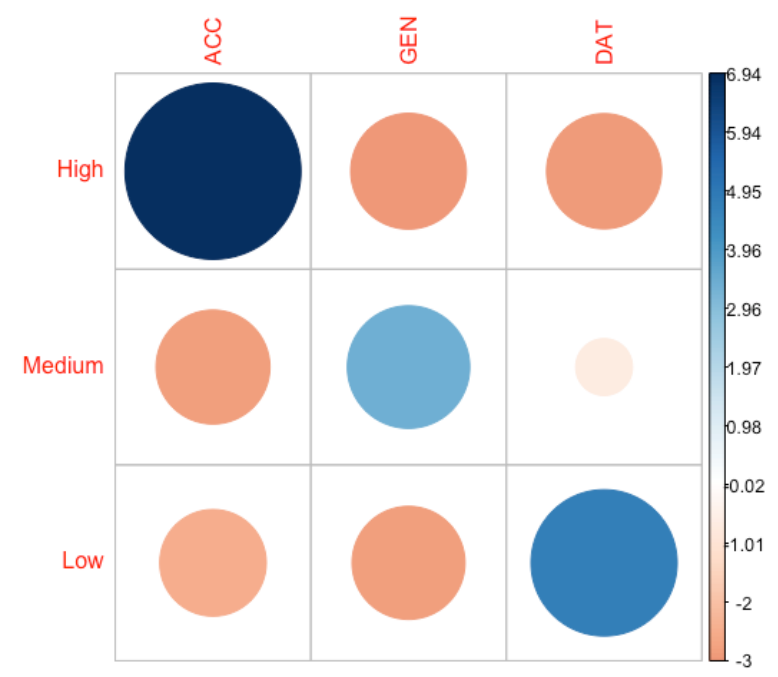

Fig. 2: Balloon Plot of Chi-Square Residuals (Transitivity Hierarchy, Revised Features)

7. Discussion and conclusions. If Luraghi's features are correct, then her Transitivity Hierarchy cannot be, and if her Hierarchy is correct, then it must be sensitive to other features than those that she proposes. As the results above demonstrate, the Affectedness Object Protorole does appear to distinguish ACC, GEN, and DAT verbs, but would correlate with a hierarchy in which DAT case-assigning verbs are more transitive than GEN case-assigning verbs. Replacing object Affectedness with the subject Volitionality, Luraghi's Hierarchy is confirmed. We therefore conclude that the latter solution is the correct. There are two additional reasons for favoring the solution provided here.

First, there is a class of verbs which can case-mark their objects with either ACC or GEN. If such verbs are intermediate between High Transitivity (HT) and Medium Transitivity (MT), as we have shown, then this optionality makes sense. However, if ACC verbs are HT and GEN verbs are Low Transitivity (LT), as would be the case if their levels of transitivity are determined by object Affectedness, then one would not expect this category to arise, since the ACC/GEN alternation would occur across non-adjacent categories. Second, we believe that Volitionality is a property which is more ontologically-salient than Affectedness, in that the volitional actions of a sentient subject are more readily observable than whether or not an object is affected. Compare examples (4) and (5), wherein the GEN object of amelein 'neglect' would be [+affected] and the DAT object of apeilēsas 'threaten' would not be so, according to Luraghi. It is not clear to us, nor to anyone with whom we've consulted, that the object of 'neglect' is necessarily affected (or more affected than the object of 'threaten'). For this reason, we conclude that the indeterminacy of Affectedness, relative to Volitionality, makes it a poor candidate for predicting transitivity.

This analysis, therefore, confirms Luraghi's ranking of ACC $>$ GEN $>$ DAT case-marking verbs within her Transitivity Hierarchy, but demonstrates COS and Volitionality (rather than COS and Affectedness) to be the determining factors. ACC case-assigning verbs are [+COS] and others are not, and among the two others, GEN case-assigning verbs are always [+volitional] and DAT case-assigning verbs are not (always).

In Classicists' discussion of Greek case, such an analysis fundamentally changes the conversation around how GEN case-assigning verbs are described. In nearly every authoritative grammar of Classical Greek, including the most recent (Boas et al. 2019), GEN case on direct objects is described as "partitive", and while there certainly are instances of partitive GEN case 
in the data, not all GEN objects are so. Where ACC and GEN case-assigning verbs are distinguished by the parameter of $[ \pm \mathrm{COS}]$, we submit that both the case assigned by both categories of verbs is "pure" and "lexically-assigned", reflecting the core meaning of the verb.

\section{References}

Bar-asher Siegal, Elitzur \& Nora Boneh. 2015. Decomposing affectedness: Truth-conditional non-core datives in Modern Hebrew. Proceedings of IATL30. MIT Working Papers in Linguistics 78. Cambridge, MA: MITWPL. http://mitwpl.mit.edu/catalog/mwpl78/.

Boas, Evert van Emde, Albert Rijksbaron, Luuk Huitink, \& Mathieu de Bakker. 2019. The Cambridge grammar of Classical Greek. Cambridge: Cambridge University Press. https://doi.org/10.1017/s2058631019000308.

Crane, Gregory R. (ed.). 2005-2019. Perseus Digital Library (Perseus 4.0). Medford, MA: Tufts University. http://www.perseus.tufts.edu (accessed December 7, 2019).

Dowty, David. 1991. Thematic proto-roles and argument selection. Language 67(3). 547-619. https://doi.org/10.1353/lan.1991.0021.

Filip, Hannah. 1996. Psychological predicates and the syntax-semantics interface. In Adele E. Goldberg (ed.), Conceptual structure, discourse, and language. 131-147. Stanford: Center for the Study of Language and Information. https://web.stanford.edu/group/cslipublications/cslipublications/site/1575860406.shtml.

Hansen, Hardy \& Gerald M. Quinn. 1992. Greek: An intensive course ( ${ }^{\text {nd }}$ revised edn.). New York: Fordham University Press. https:/www.fordhampress.com/9780823216635/greek/.

Jackendoff, Ray. 1987. The status of thematic relations in linguistic theory. Linguistic Inquiry 18(3). 369-411. https://www.jstor.org/stable/4178548.

Luraghi, Silvia. 2003. On the meaning of prepositions and cases: The expression of semantic roles in Ancient Greek. Amsterdam: John Benjamins. https://doi.org/10.1075/slcs.67.

Luraghi, Silvia. 2010. The extension of the transitive construction in Ancient Greek. Acta Linguistica Hafniensia 42. 60-74. https://doi.org/10.1080/03740463.2010.486919.

RStudio Team. 2018. RStudio: Integrated development for R. Boston, MA: RStudio. http://www.rstudio.com/.

Smyth, Herbert \& Gordon Messing (eds.). 1956. Greek grammar. Cambridge, MA: Harvard University Press. https://www.hup.harvard.edu/catalog.php?isbn=9780674362505.

Warnes, Gregory R., Ben Bolker, Lodewijk Bonebakker, Robert Gentleman, Wolfgang Huber, Andy Liaw, Thomas Lumley, Martin Maechler, Arni Magnusson, Steffen Moeller, Marc Schwartz \& Bill Venables. 2019. gplots: Various R programming tools for plotting data. R package version 3.0.1.1. https:/CRAN.R-project.org/package=gplots.

Wei, Taiyun \& Viliam Simko. 2017. R package "corrplot": Visualization of a correlation matrix (Version 0.84). https://github.com/taiyun/corrplo. 